\title{
Traffic speed deflectometer measurements at the Aurora instrumented road test site
}

\author{
C.P. Nielsen \\ Greenwood Engineering A/S, Copenhagen, Denmark \\ P. Varin \& T. Saarenketo \\ Roadscanners Ltd, Rovaniemi, Finland \\ P. Kolisoja \\ Tampere University, Tampere, Finland
}

\begin{abstract}
The results from a comprehensive set of validation measurements of the Traffic Speed Deflectometer (TSD) are presented. The measurements took place at the Aurora instrumented road test site in Muonio, Finnish Lapland, at a range of different driving speeds and road temperatures. The ability of the TSD to accurately measure pavement surface response, was validated by comparing the TSD measurements to measurements by in-situ displacement transducers. In addition, a linear viscoelastic back-calculation algorithm was applied to the TSD measurements to produce estimates of stresses and strains inside the pavement structure. These estimates were compared to measurements by in-situ transducers at the Aurora test site. The tests revealed an excellent agreement between the surface responses measured by the two systems, and a good agreement between the strains predicted from TSD measurements and the strains measured by in-situ transducers. As expected, the back-calculated moduli of the asphalt were found to increase with driving speed and decrease with temperature.
\end{abstract}

Keywords: Traffic Speed Deflectometer, TSD, Aurora, Structural condition, Instrumented pavement

\section{INTRODUCTION}

The Traffic Speed Deflectometer (TSD) sees increased use throughout the world for network level monitoring of pavement structural condition. In recent years, improved sensor technology and new analysis techniques have pushed the boundaries for what is possible with TSD measurements. Whereas the earliest TSDs were mainly suitable as screening devices, the newest generation of TSD is able to characterize structural condition at high spatial resolution and deliver high level results such as back-calculated elastic moduli and pavement strains (Nielsen 2019).

The ability of the TSD to characterize broad structural condition has earlier been validated by comparisons with multi-depth deflectometer (Velarde et al. 2016; Kannemeyer, Lategan, and Mckellar 2014), FWD (Manoharan et al. 2018; Roberts et al. 2014), and Benkelman beam measurements. However, the improved capabilities of the newest TSDs make it relevant to engage in a more detailed and ambitious program of validation. In this paper, we present results from a comprehensive measurement campaign taking place at the Aurora instrumented road test site in 
Northern Finland. The Aurora site consists of two extensively instrumented road sections with transducers for measuring surface displacement and surface acceleration in vertical direction, AC layer horizontal strain, base layer vertical strain, and vertical stress in the base layer (Kolisoja et al. 2019). The comparisons between the TSD measurements and the transducer measurements take place in two stages. In the first stage, the ability of the TSD to measure the surface response accurately, is validated by a direct comparison of the surface measurements from both systems. The second stage focuses on derived values, and thus constitute a test of the combined measurement and data analysis system. In this stage, back-calculated pavement strains are compared with the strains measured in-situ by the strain transducers. To assess the importance of driving speed and temperature, the measurements took place at a range of driving speeds and at different times of day.

\section{MEASUREMENT DESCRIPTION}

Two measurement systems were used in this study to provide independent readings of the pavement response. The Traffic Speed Deflectometer is a nondestructive pavement evaluation device, which uses laser Doppler vibrometers to measure the pavement response to a moving 10 tonne axle. The Doppler lasers measure the deflection velocity of the pavement surface at several points on the line passing between the center of the truck's twin tires. Combining this with a measurement of the driving speed, the slope of the deflection basin is recovered. See Refs. (Krarup et al. 2006; Nielsen 2019) for more information.

The Aurora instrumented road test site is part of an open testing ecosystem of intelligent transport and infrastructure solutions launched by the Finnish Transport Infrastructure Agency (FTIA). It includes two road sections extensively instrumented for monitoring both structural responses and condition of these road sections. The Aurora 1 site is located on a stiff subgrade soil, where an old road structure with good bearing capacity was strengthened by laying a new $50 \mathrm{~mm}$ overlay on top of the old pavement leading to $120 \mathrm{~mm}$ of bound layers. The Aurora 2 site is located in softer subgrade with deformation problems in the old pavement structure. For this reason, the top part of the old pavement structure was mixmilled and a bound base and $\mathrm{AC}$ wearing course, altogether $90 \mathrm{~mm}$, was paved on top of the mixmilled base. A special feature in the base course material at the Aurora test site is that it has very high suction properties. The transducers at the Aurora test site operate with a sampling rate of $1 \mathrm{kHz}$ and they allow us to probe the true pavement behavior, both at the surface and inside the pavement structure. To reduce noise, the transducer outputs were filtered with a forward-backward third order Butterworth filter. The cutoff frequency for the filter was chosen to correspond to a wavelength of $40 \mathrm{~cm}$ at the driving speed of the TSD.

The measurements took place on the $6^{\text {th }}$ and $7^{\text {th }}$ of July 2020 at the Aurora instrumented road test site in Finnish Lapland. Over the course of the measurements the air temperature ranged between $12.5^{\circ} \mathrm{C}$ and $20.5^{\circ} \mathrm{C}$, and the road surface temperature ranged between $13{ }^{\circ} \mathrm{C}$ and $24^{\circ} \mathrm{C}$. To test the effect of driving path, 11 measurements were made with a constant driving speed of $80 \mathrm{~km} / \mathrm{h}$ and with varying distances to the road side. To test the effect of driving speed, 3 additional measurements were made with each of the driving speeds $10 \mathrm{~km} / \mathrm{h}, 20 \mathrm{~km} /$ $\mathrm{h}, 40 \mathrm{~km} / \mathrm{h}$, and $80 \mathrm{~km} / \mathrm{h}$. These measurements were made in the evening on the $6^{\text {th }}$ with a road surface temperature around $23{ }^{\circ} \mathrm{C}$. In the morning the next day, another 3 measurements were made with each of the driving speeds $20 \mathrm{~km} / \mathrm{h}, 40 \mathrm{~km} / \mathrm{h}$, and $80 \mathrm{~km} / \mathrm{h}$. This time with a road surface temperature around $14.5^{\circ} \mathrm{C}$. Also, a single measurement with a driving speed of $5 \mathrm{~km} /$ $\mathrm{h}$ was made. The approximate lateral position of the TSD was determined using vertical accelerometers located with $10 \mathrm{~cm}$ spacing across the driving path. The last bit of alignment was done by correlating the back-calculated TSD results with the transducer measurements. 


\section{COMPARING SURFACE DISPLACEMENT MEASUREMENTS}

The Aurora 2 site has three surface displacement transducers mounted at different lateral positions on the road. By differentiating the displacements with respect to time, the deflection velocities can be found. This enables a direct comparison between the deflection velocities measured by the TSD and the deflection velocities measured by the in-situ displacement transducers. This comparison is, however, dependent on the driving path of the TSD; only for the cases where the center of the twin tires passes directly over a displacement transducer, should we expect a good agreement between the two systems. In Figure 1 two examples of deflection velocities measured by the TSD and the displacement transducers are shown. By using the TSD driving speed $v_{0}$, the Aurora recording times are converted to positions $x=v_{0} t$. This allows for a direct comparison of the two systems on the same axis. Here, and in the remainder of the paper, the reported TSD measurements were averaged over a distance of 2 meters. The black dots indicate the velocities measured by the TSD, and the dashed colored lines show the velocities measured by the displacement transducers. Each line is labeled by the transducer's lateral distance to the path measured by the TSD. It is seen that the displacement transducers close to the TSD path have an excellent agreement with the velocities measured by the TSD.
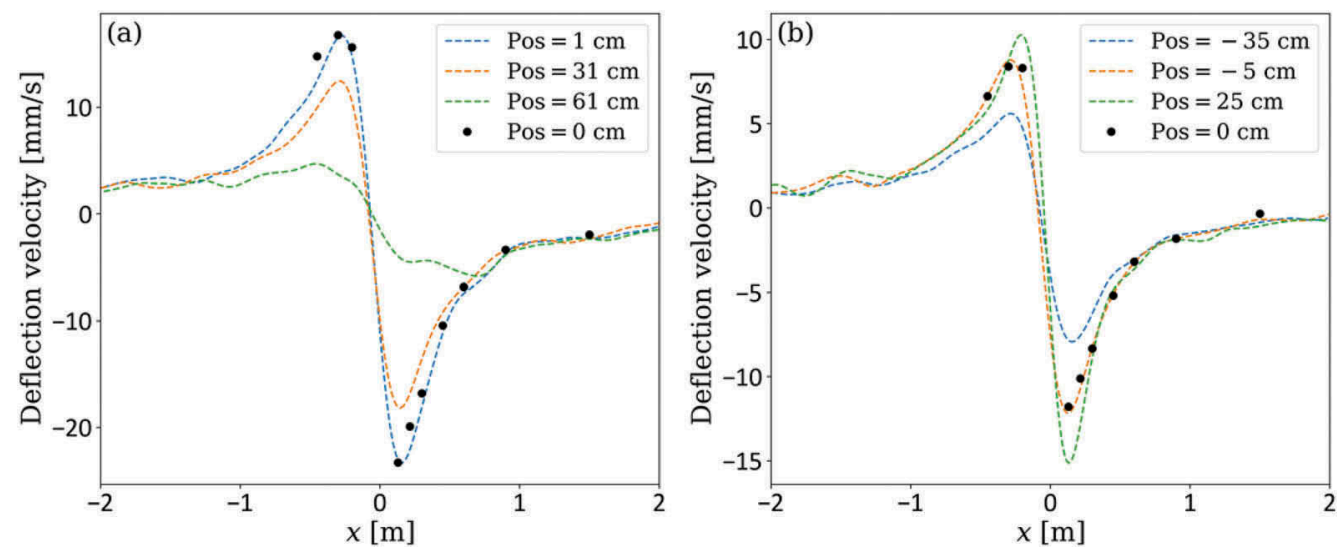

Figure 1. Deflection velocities measured by the TSD (black dots) and measured by the displacement transducers (dashed lines). (a): measurement from day one with a driving speed of $80 \mathrm{~km} / \mathrm{h}$. (b): measurement from day two with driving speed of $20 \mathrm{~km} / \mathrm{h}$.

\section{BACK-CALCULATION}

The TSD measurements were processed using a linear viscoelastic back-calculation algorithm developed specifically for the TSD. By taking material viscoelasticity and damping into account the algorithm is able to capture the asymmetric nature of the deflection basin under a moving load. The outputs of the algorithm are back-calculated layer moduli, as well as displacements, stresses and strains at any point in the pavement structure. See Ref. (Nielsen 2019) for details.

The layer thicknesses used in the back-calculation were based on GPR measurements, probing with a sounding rod, and visual inspection of the upper layers. The layer thicknesses are shown in Table 1. For the purposes of back-calculation the stiff moraine layer is implemented as bedrock. 
Table 1. Pavement layers at the two Aurora sites.

\begin{tabular}{ll}
\hline Aurora 1 & Aurora 2 \\
\hline $12 \mathrm{~cm}$ asphalt concrete (AC) & $9 \mathrm{~cm}$ asphalt concrete (AC) \\
$20 \mathrm{~cm}$ base course & $25 \mathrm{~cm}$ base course \\
$30 \mathrm{~cm}$ sub-base course & $30 \mathrm{~cm}$ sub-base course \\
$50 \mathrm{~cm}$ coarse grained material & $336 \mathrm{~cm}$ sandy material and subgrade \\
Stiff moraine & Stiff moraine \\
\hline
\end{tabular}

For each measurement point, the back-calculation algorithm provides values for the complex modulus in each of the four pavement layers. The modulus of the AC layer is of particular interest, since this is expected to change with driving speed and temperature. In Figure 2 the real part of the back-calculated AC modulus is plotted versus driving speed at the Aurora 1 and Aurora 2 sites. The red set of measurements were made in the evening of day one, with a road surface temperature of $23^{\circ} \mathrm{C}\left(1{ }^{\circ} \mathrm{C}\right)$, and the blue set of measurements were made in the morning of day two, with a road surface temperature of $14.5^{\circ} \mathrm{C}\left(1{ }^{\circ} \mathrm{C}\right)$. It is seen that the back-calculated moduli increase with driving speed and decrease with temperature. This agrees with standard AC behavior, and supports our assumption that the back-calculation algorithm finds the true AC layer moduli.
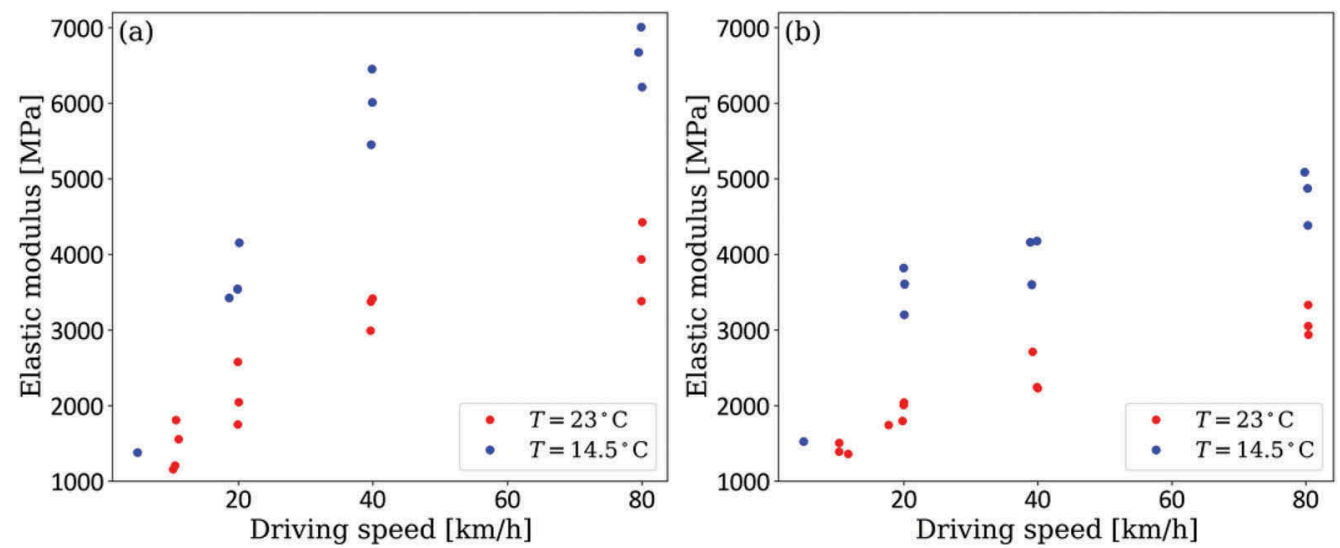

Figure 2. Back-calculated elastic moduli of the AC layer plotted versus driving speed for two different temperatures at the Aurora 1 site (a) and the Aurora 2 site (b).

\subsection{Back-calculated field values}

The back-calculated deflection velocities, strains, and stresses can be directly compared to the values measured by the various transducers. In the following, back-calculated and measured field values are shown for two of the measurements at Aurora 2. The first measurement was made on day one with a driving speed of $80 \mathrm{~km} / \mathrm{h}$ and the other was made on day two with a driving speed of $20 \mathrm{~km} / \mathrm{h}$.

In Figure 3 examples of back-calculated and measured deflection velocities are shown for the two measurements at Aurora 2. The black dots show the deflection velocities measured at the centerline of the twin tires, the dashed lines show the velocities measured by the transducers, and the full lines show the back-calculated deflection velocities evaluated at the 
position of the transducers. Each line is labeled by the transducer's lateral distance to the path measured by the TSD. In Figure 3 (b) the deflection velocity obtained from the transducer with the green dashed line is seen to exceed the deflection velocity back-calculated from TSD measurements. The reason for this is probably that the measurement is made under one of the tires, and therefore the details of the tire footprint become important. In the current implementation, the back-calculation algorithm treats each tire as a circular load of radius $10 \mathrm{~cm}$. For most purposes this is sufficiently realistic to yield good results, but it is expected to lead to discrepancies very close to or under the tire.
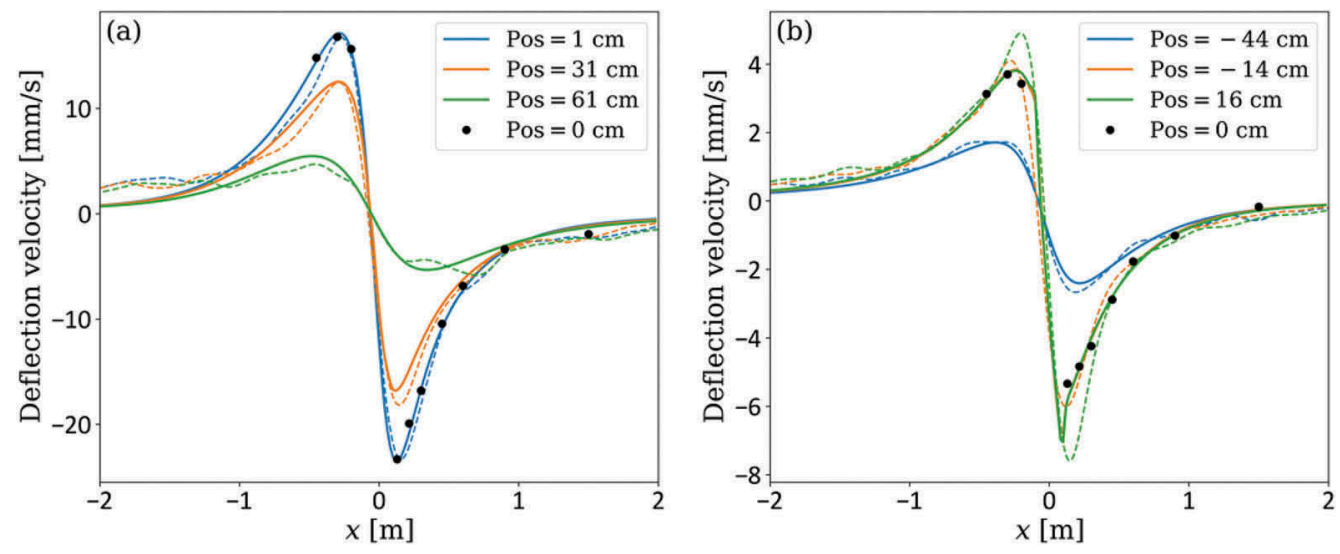

Figure 3. Deflection velocities measured by the TSD (black dots), back-calculated from TSD measurements (full lines), and measured by the displacement transducers (dashed lines). (a): measurement from day one with a driving speed of $80 \mathrm{~km} / \mathrm{h}$. (b): measurement from day two with driving speed of $20 \mathrm{~km} / \mathrm{h}$.

In Figure 4 examples of back-calculated and measured longitudinal strains in the bottom of the AC layer are shown. There is seen to be good agreement between the back-calculated and measured strains. In Figure 5 examples of back-calculated and measured transversal strains in the bottom of the AC layer are shown. The agreement between measured and back-calculated strains is quite good in Figure 5 (b), but in Figure 5 (a) there are significant differences between the predicted and measured strains. The measurements in Figure 5 (a) are made close to or under the tire, so the observed discrepancies could be due to the assumption of a circular tire footprint. In Figure 6 examples of back-calculated and measured vertical strains in the base layer are shown. There is seen to be good agreement between the predicted and measured values. In Figure 7 examples of back-calculated and measured vertical pressures in the base layer are shown. To improve readability of the figure, only results from 4 of the 8 installed pressure transducers are plotted. These sensors were located at a depth of $280 \mathrm{~mm}$ (blue and orange lines) and $180 \mathrm{~mm}$ (green and red lines). The agreement between the predicted and measured values is seen to be quite poor, with some of the pressures differing by more than a factor of two. In light of the good agreement between the base layer strains in Figure 6, it is somewhat surprising to observe so large discrepancies in the base layer stresses. This issue is discussed further in the next section.

\subsection{Comparison of peak values}

In the preceding section the back-calculated and measured field values were compared in detail for two of the measurements. In order to take all 34 measurements into account, a more 

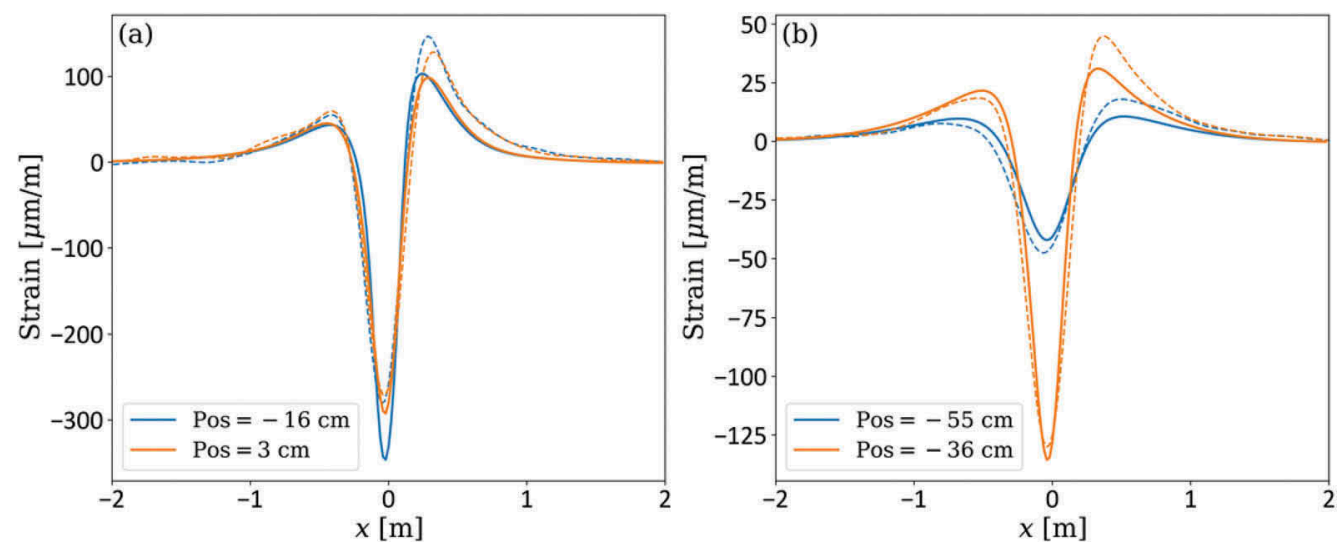

Figure 4. Longitudinal strains in bottom of AC layer back-calculated from TSD measurements (full lines) and measured by strain gauges (dashed lines). (a): measurement from day one with a driving speed of $80 \mathrm{~km} / \mathrm{h}$. (b): measurement from day two with driving speed of $20 \mathrm{~km} / \mathrm{h}$.
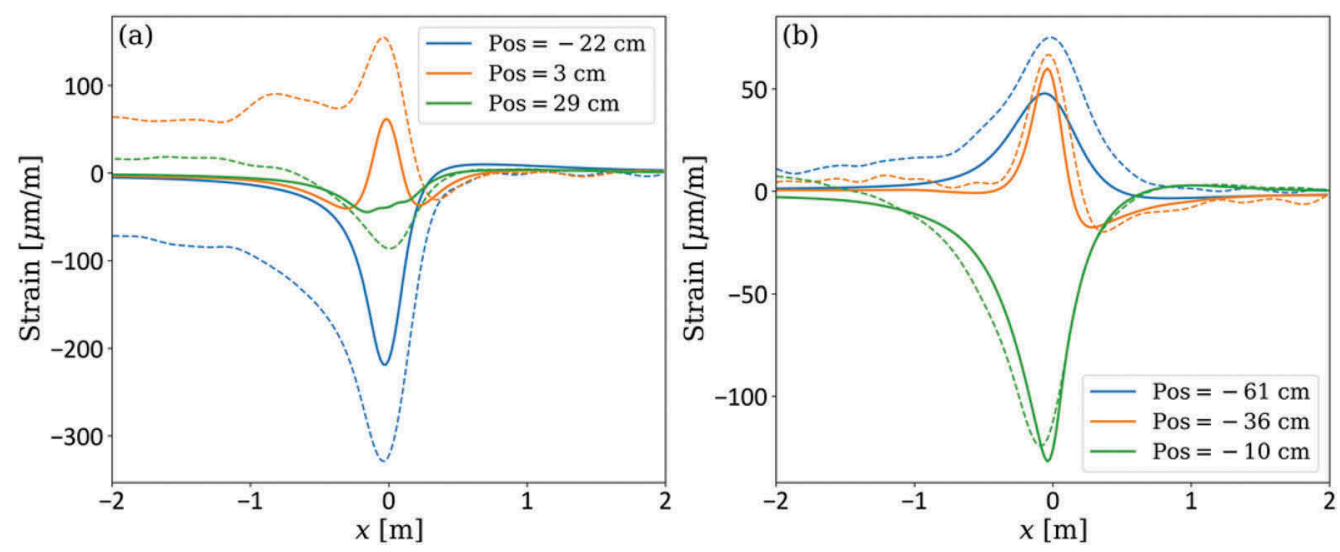

Figure 5. Transversal strains in bottom of AC layer back-calculated from TSD measurements (full lines) and measured by strain gauges (dashed lines). (a): measurement from day one with a driving speed of $80 \mathrm{~km} / \mathrm{h}$. (b): measurement from day two with driving speed of $20 \mathrm{~km} / \mathrm{h}$.

compact way of comparing data is applied in this section. For each measurement, the peak values of the measured fields are extracted and plotted versus the corresponding peak values of the back-calculated fields. For the deflection velocity, both the positive and the negative peaks are plotted.

In Figure 8 (a) the peak deflection velocities are plotted together with the line of equality. There is seen to be an excellent agreement between the peak velocities measured by the two systems. In Figure 8 (b) the peak longitudinal strains in the bottom of the AC layer are plotted. The relation between the TSD strains and the Aurora strains is better described by a line with slope 0.85 (grey) than by the line with slope 1 (black). This scale error is likely caused by small deviations in the AC layer thickness or sensor depth. For instance, a difference in AC layer thickness of just $13 \mathrm{~mm}$ is enough to explain the observed scale error.

In Figure 9 (a) the peak transversal strains in the bottom of the AC layer are plotted. Some amount of scatter around the line of equality is observed, but in general the agreement between the two systems is reasonably good. In Figure 9 (b) the peak vertical strains in the 

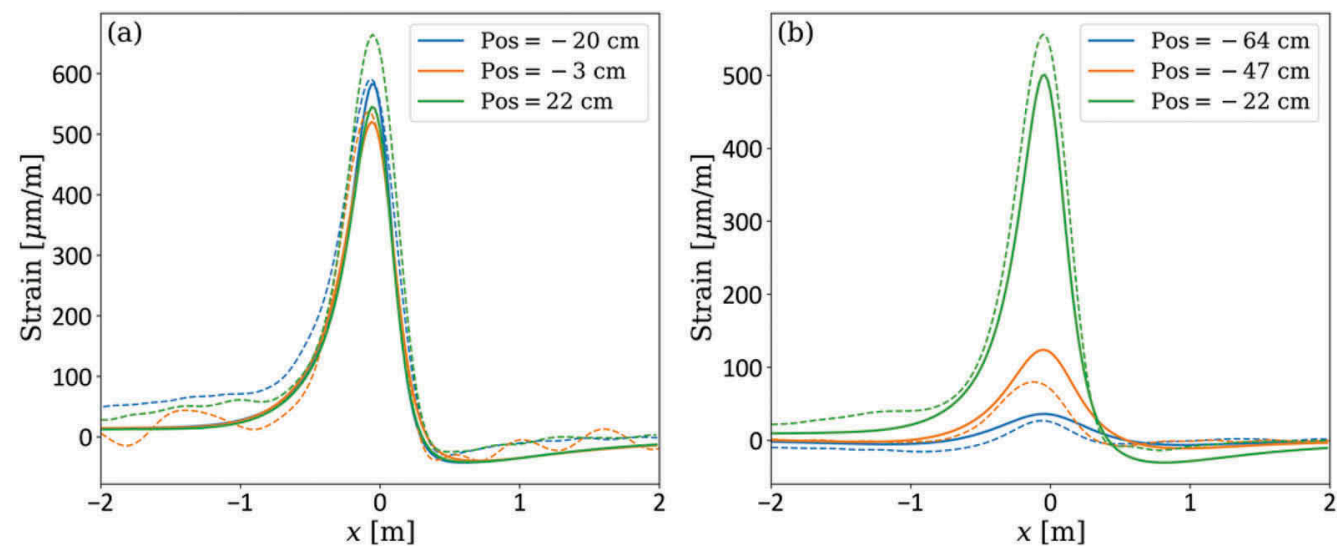

Figure 6. Vertical strains in base layer back-calculated from TSD measurements (full lines) and measured by strain transducers (dashed lines). (a): measurement from day one with a driving speed of $80 \mathrm{~km} /$ h. (b): measurement from day two with driving speed of $20 \mathrm{~km} / \mathrm{h}$.
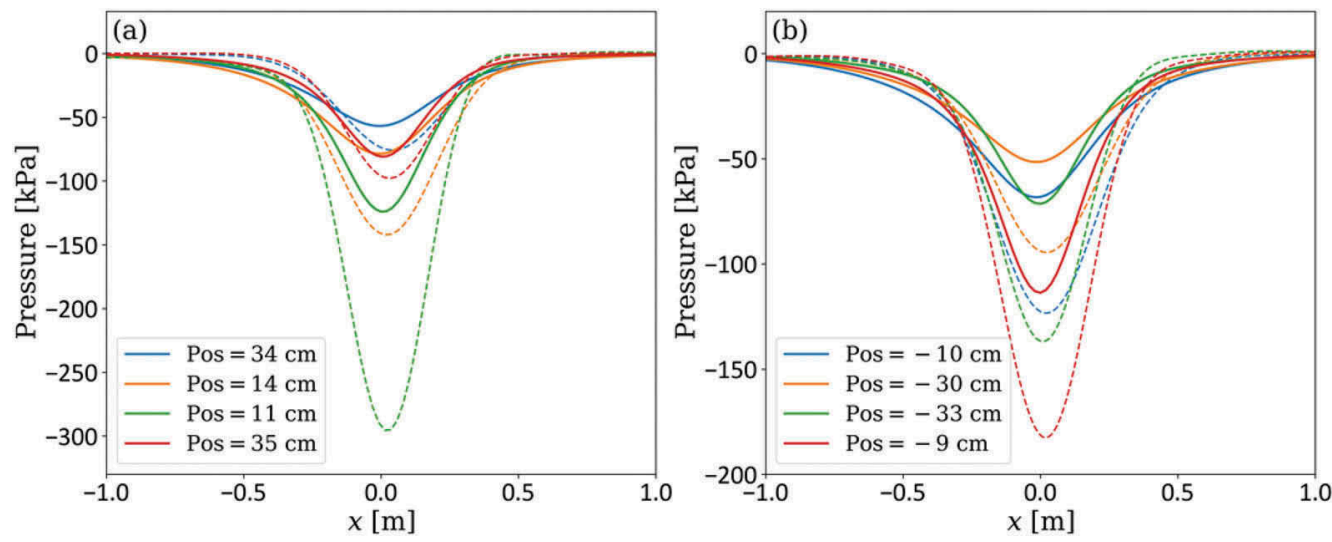

Figure 7. Vertical pressure in base layer back-calculated from TSD measurements (full lines) and measured by pressure transducers (dashed lines). (a): measurement from day one with a driving speed of $80 \mathrm{~km} /$ h. (b): measurement from day two with driving speed of $20 \mathrm{~km} / \mathrm{h}$.

base layer are plotted. At low strains, the TSD strains are seen to be larger than the corresponding Aurora strains. In general, the agreement between the systems is, however, quite good.

In Figure 10 (a) the peak vertical pressures in the base layer are plotted. The data points are seen to fall far from the line of equality, and in general the agreement between the two systems is poor. This is consistent with the finding in Figure 7, where the detailed pressure distributions were considered. As was previously noted, it is surprising to observe so large discrepancies in the stresses, when all the strain components exhibit a good agreement between the two systems. The likely reason for this discrepancy is nonlinear behavior of the unbound layers, something which is not accounted for in the linear viscoelastic pavement model used to back-calculate the TSD measurements. The simplest model for unbound layer nonlinearity is the so-called " $\mathrm{k}$-theta" model, originally suggested by Brown and Pell (1967). It states that the resilient modulus $M_{\mathrm{r}}$ depends on the sum of principal stresses $\theta$ as, 

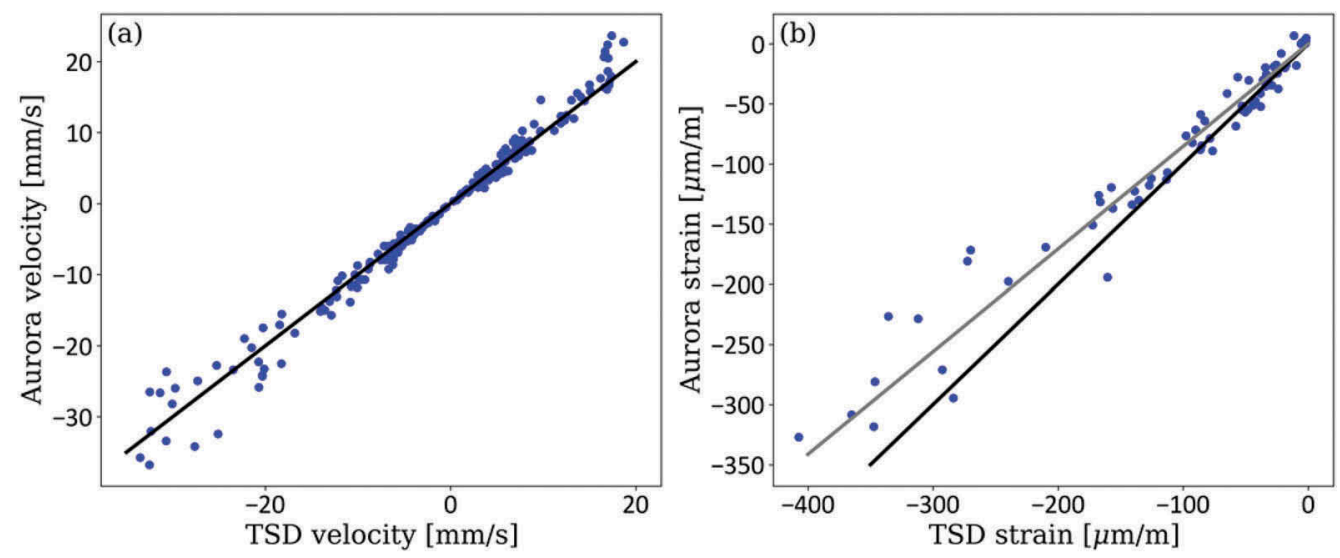

Figure 8. (a): Aurora peak deflection velocities plotted versus the TSD peak deflection velocities. The line of equality is shown in black. (b): Aurora peak longitudinal strains plotted versus TSD peak longitudinal strains. The line of equality is shown in black and a line with slope 0.85 is plotted in grey.

$$
M_{\mathrm{r}}=k_{1} P_{\mathrm{a}}\left(\frac{\theta}{P_{\mathrm{a}}}\right)^{k_{2}}
$$

where $k_{1}$ and $k_{2}$ are material parameters, and the atmospheric pressure $P_{\mathrm{a}}$ is used here to make Equation 1 dimensionally consistent. Since the back-calculated strains have a good agreement with the measured strains, it should be possible to recover the pressure $\sigma_{z z}^{\text {Aurora }}$ from the resilient modulus and the back-calculated strain $E_{z z}^{\mathrm{TSD}}$,

$$
\sigma_{z z}^{\text {Aurora }} \propto M_{\mathrm{r}} \varepsilon_{z z}^{\mathrm{TSD}} \propto \theta^{k_{2}} \sigma_{z z}^{\mathrm{TSD}} \propto\left(\sigma_{z z}^{\mathrm{TSD}}\right)^{1+k_{2}}
$$

where the last expression makes the simplifying assumption that the sum of principal stresses $\theta$ is proportional to $\sigma_{z z}^{\mathrm{TSD}}$. In Figure 10 (b) the Aurora pressure is plotted versus the back-calculated pressure in a log-log plot. It is seen that the points are well approximated by a power law with exponent 1.5 (red line). This corresponds to a $k_{2}$ value of 0.5 , which agrees with the values reported in the literature (Kolisoja 1997). It is noted, however, that for high pressures, the data points in Figure 10 (b) start to deviate from the simple power law. This implies that the $\mathrm{k}$-theta model is not able to describe the nonlinear unbound material behavior correctly at high deviator stress levels.

The arguments given above indicate, that there is a considerable difference between the subgrade modulus determined by the linear viscoelastic back-calculation and the true resilient modulus of the subgrade. Nevertheless, the estimated and measured subgrade strains are seen to agree reasonably well. The reason for this is, that the subgrade strain has to be consistent with the measured surface deflection. So even though the subgrade modulus is poorly estimated, the estimated subgrade strain cannot deviate too much from the true value. This is in line with the findings in (Nielsen 2020), where it was shown that good estimates of the pavement strains can be obtained from knowledge of the deflection basin, even when the elastic moduli are not known. 

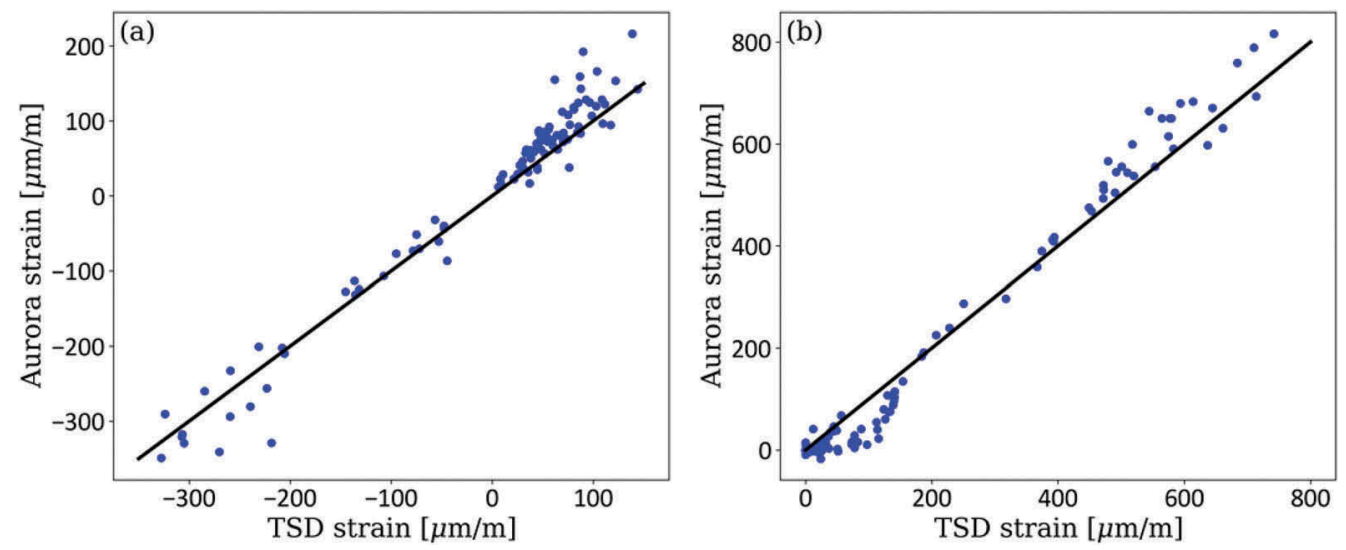

Figure 9. (a): Aurora peak transversal strains plotted versus TSD peak transversal strains. The line of equality is shown in black. (b): Aurora peak vertical base course strains plotted versus TSD peak vertical base course strains. The line of equality is shown in black.
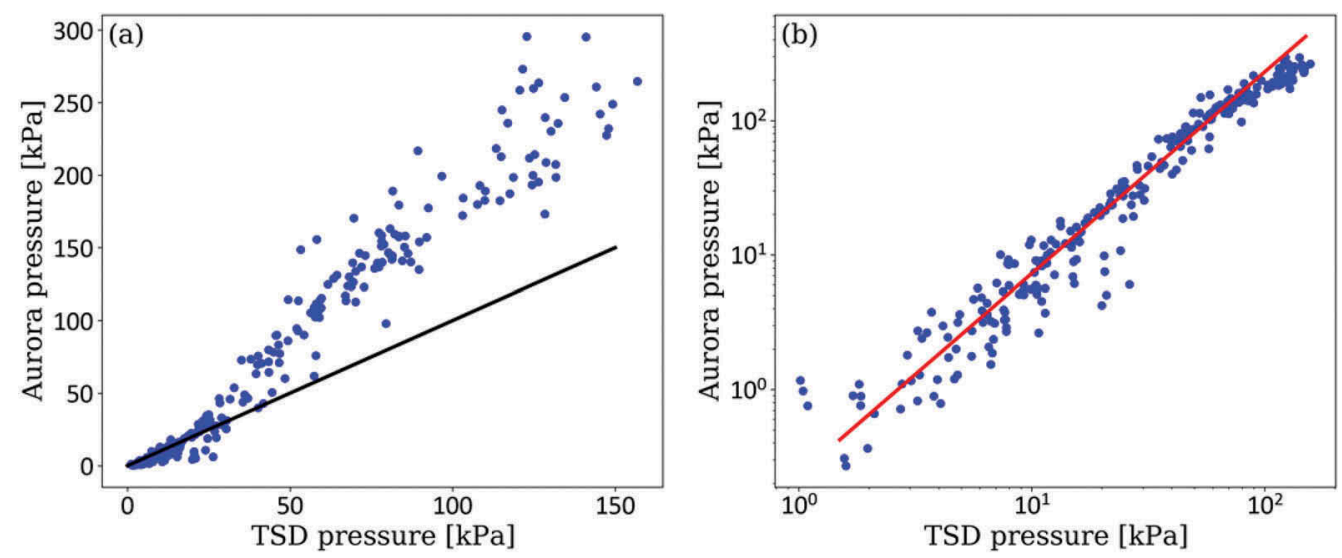

Figure 10. (a): Aurora peak vertical pressures plotted versus the TSD peak vertical pressures. The line of equality is shown in black. (b): Same as (a), but plotted with logarithmic axes. The red line is a power law with exponent 1.5 .

\section{CONCLUSION}

The ability of the TSD to assess pavement response under a moving load, was evaluated by comparison with in-situ transducers at the Aurora instrumented road test site. The pavement deflection velocities measured by the TSD were found to have an excellent agreement with the deflection velocities measured by the in-situ displacement transducers. Estimates of stresses and strains inside the pavement structure were obtained by applying a linear viscoelastic backcalculation algorithm to the TSD measurements. There was found to be a good agreement between measured and back-calculated horizontal strains in the bottom of the AC layer and vertical strains in the bottom of the base layer. In contrast, there was a poor agreement between measured and back-calculated pressures in the base layer. This was found to be due to stress hardening of the base layer material, something which was not accounted for in the linear viscoelastic pavement model. In agreement with standard rheological models for 
asphalt, the back-calculated moduli of the AC layer were found to increase with driving speed and decrease with temperature.

\section{ACKNOWLEDGEMENTS}

The authors would like to thank Nuutti Vuorimies and Antti Akkanen from Tampere University for valuable assistance during the measurement campaign.

\section{REFERENCES}

Brown, S. F., and P. S. Pell. 1967. An Experimental Investigation of the Stresses, Strains and Deflections in a Layered Pavement Structure Subjected to Dynamic Loads, In Proceedings of the 2nd International Conference on Structural Design of Asphalt Pavements, 487-504.

Kannemeyer, L, W Lategan, and A Mckellar. 2014. Verification of Traffic Speed Deflectometer Measurements Using Instrumented Pavements in South Africa, 1-29.

Kolisoja, P. 1997. Resilient Deformation Characteristics of Granular Materials, Tampere University of Technology.

Kolisoja, P, N Vuorimies, A Kurki, and T Saarenketo. 2019. Open Structural Monitoring Data from Two Extensively Instrumented Road Sections - Case Aurora, In Proceedings of the XVII ECSMGE-2019

Krarup, J., S. Rasmussen, L. Aagaard, and P. G. Hjorth. 2006. Output from the Greenwood Traffic Speed Deflectometer, In Paper Presented to 22nd ARRB Conference. Canberra, Australia.

Manoharan, S., G. Chai, S. Chowdhury, and A. Golding. 2018. A Study of the Structural Performance of Flexible Pavements Using Traffic Speed Deflectometer, Journal of Testing and Evaluation 46 (3): $1280-89$.

Nielsen, C.P. 2019. Visco-Elastic Back-Calculation of Traffic Speed Deflectometer Measurements. Transportation Research Record.

Nielsen, C.P. 2020. Deriving Pavement Deflection Indices from Layered Elastic Theory. Transportation Research Record.

Roberts, Jon, Ulysses Ai, Tyrone Toole, and Tim Martin. 2014. Traffic Speed Deflectometer Data Review and Lessons Learnt.

Velarde, J. A., S. Rocha, S. Nazarian, G. Rada, S. Thyagarajan, and R. V. Siddharthan. 2016. Use of Embedded Sensors to Evaluate Performance of Traffic Speed Deflection Devices, Journal of Testing and Evaluation 45 (4): 1316-25. 